\title{
SHORT REPORT \\ Genotyping of Leptospira directly in urine samples of cattle demonstrates a diversity of species and strains in Brazil
}

\author{
C. HAMOND ${ }^{1}$, C. P. PESTANA ${ }^{2}$, M. A. MEDEIROS ${ }^{2}$ AND W. LILENBAUM ${ }^{1}$ * \\ ${ }^{1}$ Laboratory of Veterinary Bacteriology, Department of Microbiology and Parasitology, Universidade Federal \\ Fluminense, Niterói, RJ, Brazil \\ ${ }^{2}$ Bio-Manguinhos, Oswaldo Cruz Foundation, Brazilian Ministry of Health, Rio de Janeiro, RJ, Brazil
}

Received 10 December 2014; Final revision 28 May 2015; Accepted 29 May 2015;

first published online 16 June 2015

\section{SUMMARY}

The aim of this study was to identify Leptospira in urine samples of cattle by direct sequencing of the $\sec Y$ gene. The validity of this approach was assessed using ten Leptospira strains obtained from cattle in Brazil and 77 DNA samples previously extracted from cattle urine, that were positive by PCR for the genus-specific lipL32 gene of Leptospira. Direct sequencing identified 24 $(31 \cdot 1 \%)$ interpretable $\sec Y$ sequences and these were identical to those obtained from direct DNA sequencing of the urine samples from which they were recovered. Phylogenetic analyses identified four species: L. interrogans, L. borgpetersenii, L. noguchii, and L. santarosai with the most prevalent genotypes being associated with $L$. borgpetersenii. While direct sequencing cannot, as yet, replace culturing of leptospires, it is a valid additional tool for epidemiological studies. An unexpected finding from this study was the genetic diversity of Leptospira infecting Brazilian cattle.

Key words: Leptospira, $\sec Y$, urine sample.

Leptospira are the causative agents of leptospirosis and are zoonotic pathogens with a worldwide distribution. Leptospirosis is most prevalent in countries with subtropical or tropical climates which favour the survival of the organisms [1]. Leptospiral infection in cattle causes reproductive failure (abortion, embryonic death, stillbirth and infertility) and decreased milk production [2]. Importantly, infection represents a significant disease risk for farmers [3].

Cattle may become asymptomatic temporary or permanent reservoirs and shed leptospires in their urine $[2,4]$ and genital tract discharges [3]. They can also serve as maintenance hosts for Leptospira borgpetersenii

\footnotetext{
* Author for correspondence: Dr W. Lilenbaum, Department of Microbiology and Parasitology, Universidade Federal Fluminense, Niterói, RJ, Brazil.

(Email: mipwalt@vm.uff.br)
}

serovar (sv) Hardjo strain Hardjobovis as well as for L. interrogans sv Hardjo strain Hardjoprajitno [1, 4]. Leptospira can be cultured from the urine of animals, but due to their fastidious growth properties, this method is not used routinely [3] and diagnosis is most commonly performed by serological assay (microscopic agglutination test; MAT) or polymerase chain reaction (PCR) amplification of genus-specific genes which have high sensitivity and specificity for the detection of leptospiral DNA in clinical samples. However, PCR does not identify the organisms to the serovar level and is therefore not widely used in epidemiological studies [5].

When culture isolates are not available, typing by sequencing of the PCR amplified housekeeping gene $\sec Y$ can be used directly on biological samples [5]. The sec $Y$ gene encodes a preprotein translocase and is located within the $\mathrm{S} 10-s p c-\alpha$ locus containing 
genes for ribosomal proteins. The gene consists of alternating conserved and variable regions, with sufficient sequence heterogeneity to enable phylogenetic classification of Leptospira [6, 7]. Direct sequencing of $\sec Y$ has been successfully used for the identification of Leptospira in human blood, and species identification in different animal hosts [5, 6], but to our knowledge it has not been utilized for biological samples from cattle. Epidemiological studies on bovine leptospirosis are scarce, mainly due to the difficulties in obtaining isolates. The aim of the present study was to identify Leptospira from urine samples of cattle by PCR amplification of the $\sec Y$ gene and subsequent sequence analysis.

Ten Leptospira strains were obtained from cattle in Brazil. Six $L$. santarosai and two $L$. noguchii were from the Leptospires Collection of Veterinary Bacteriology Laboratory of Universidade Federal Fluminense; one L. interrogans from the Technology Development Center (CDTec), Federal University of Pelotas (UFPel), and one L. interrogans from the Department of Preventive Veterinary Medicine and Animal Health, University of São Paulo (USP).

A total of 77 DNA samples previously extracted from urine of cattle, which were Leptospira positive by PCR amplification of lipL32 were used for PCR-sec $Y$ sequencing. Cows originating from diverse herds in the region and with no apparent clinical signs of disease were randomly selected in a slaughterhouse located near Rio de Janeiro, Brazil. Of the 77 DNA samples, eight were from the original urine samples that yielded pure cultures of L. santarosai (KP263062, KP263063， KP263064， KP263067, KP263068, KP263069) and L. noguchii (KP263061, KP263066).

The sec $Y$ gene was partially amplified by PCR with primers and protocols as described previously $[5,8]$. The amplicons were sequenced in both directions using Big Dye Terminator $3 \cdot 1$ cycle sequencing kit (Applied Biosystems, USA) on ABI 3500 XL Genetic Analyzer (Life Technologies, USA). All molecular epidemiological data were stored and analysed with DNAStar Software Lasergene version 5.05 (DNASTAR, USA). Sequences were compared to ten available reference strains (NCBI) of four Leptospira species (L. interrogans, $L$. noguchii, $L$. santarosai, $L$. borgpetersenii). The phylogenetic analysis was performed with MEGA $5 \cdot 2$ software (www.megasoftware.net) using the neighbourjoining method and phylogenetic trees were constructed using the Tamura-Nei model; the reliability of the branches were validated by the generation of 1000 'bootstrap' replicates.
Of the 77 DNA samples tested, 55 (71.4\%) were PCR positive for the $\sec Y$ gene and 24/55 (43.6\%) PCR products gave interpretable sequences. An additional nine sequences derived from culture isolates made a total of 33 analysed sequences. Non-interpretable sequences were as classified by the analysis software. Importantly, identical $\sec Y$ sequences in all the eight cases of L. santarosai and L. noguchii were observed in DNA extracted from the Leptospira isolate and the urine sample from which it was recovered (Fig. 1). Of the 24 analysed sequences, six $(25 \%)$ were identified as L. santarosai, ten $(41.7 \%)$ as $L$. borgpetersenii, two $(8 \cdot 3 \%)$ as $L$. noguchii and six $(25 \%)$ as $L$. interrogans (Supplementary material). Sequencing of $\sec Y$ fragments distinguished four clusters (here defined as homology $>95 \%$ and bootstraps ranging from $70 \%$ to $99 \%$ ) by species.

Sequencing of $\sec Y$ fragments of $L$. santarosai distinguished nine sequences grouped into a single cluster. Compared to the reference strains, two L. santarosai (U152, U278) displayed homology with $L$. santarosai sv Bananal. By contrast, the remainder were distinct from any of the reference strains. The two $L$. noguchii sequences were grouped in a single cluster and displayed homology with L. noguchii sv Myocastoris (Fig. 1).

Alignment of $\sec Y$ for $L$. interrogans revealed six sequences in one cluster. One group comprised three urine samples (U5, U167, U225) and displayed homology with the serovar Canicola isolate from São Paulo (USP), while the other (U16, U85, U193) was homologous with the serovar Australis isolate from southern Brazil (UFPel).

Ten sequences were identified as $L$. borgpetersenii which displayed homology with $L$. borgpetersenii sv Hardjobovis. According to the similarity level, sequences fell into three groups; two of these displayed $97 \cdot 2 \%$ identity (U17, U183, U277, U291, U146, U275) and (U83, U215), while the third (U98, U194) displayed $>99 \%$ identity to L. borgpetersenii sv Hardjobovis.

Using the direct $\sec Y$ sequencing method, we were able to infer the species and genotypes without the need to culture the pathogen; importantly, sec $Y$ sequences from urine samples and the respective Leptospira isolates from these urine samples yielded identical results, thus validating the method. In addition to PCR detection, sequencing of the $\sec Y$ gene allows species identification in bovine leptospiral infection, and should be utilized in other regions and environments. The observed non-concordance 


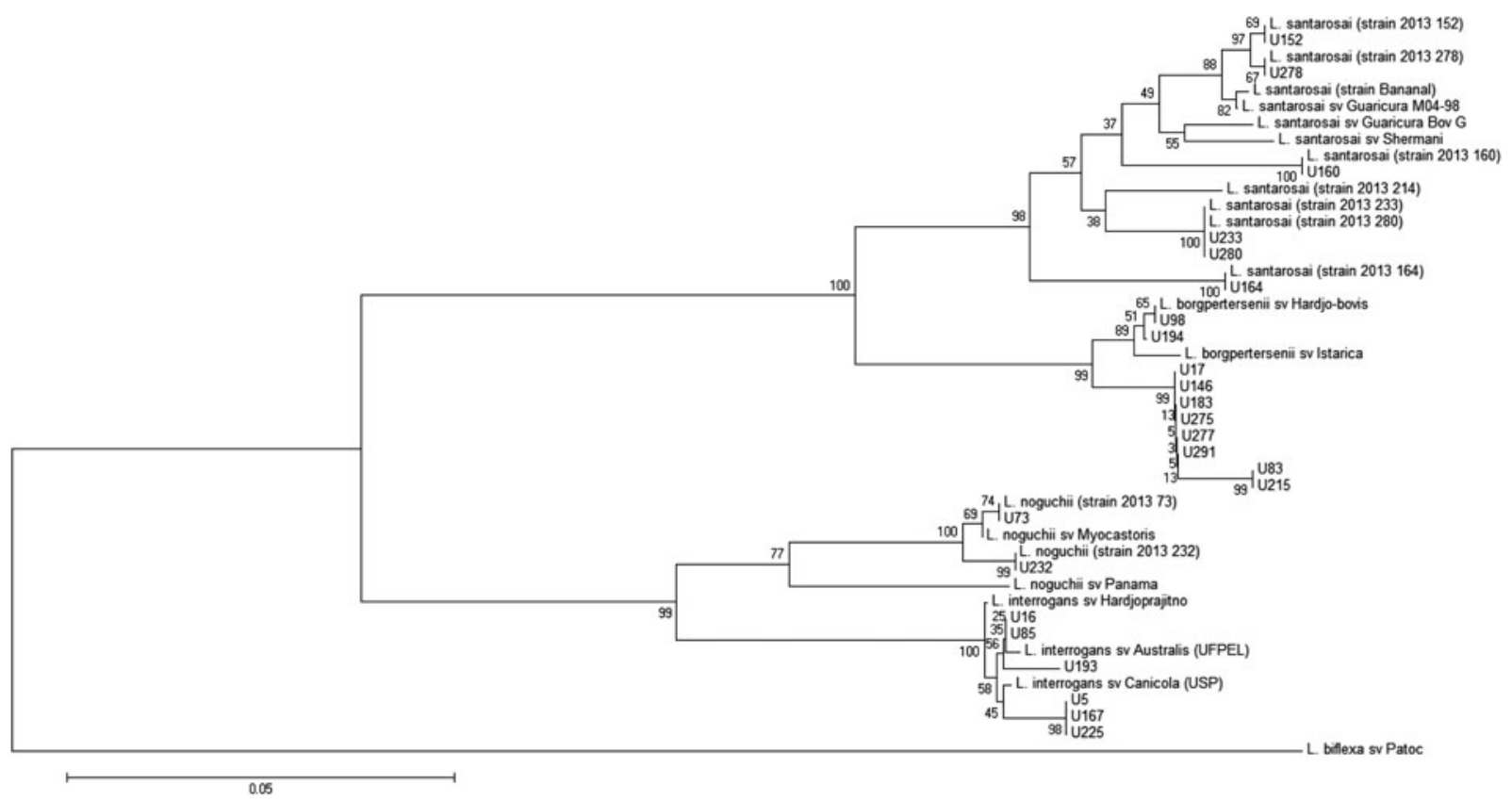

Fig. 1. Phylogenetic tree of Leptospira infecting cattle in Brazil. The phylogenetic tree was drawn using the Tamura-Nei model. Reliability of the branches was validated by the generation of 1000 'bootstrap' replicates.

between the positivity of lipL32-PCR and $\sec Y$-PCR for 22 DNA samples raises the possibility of falsepositive results from lipL32-PCR. However, this discrepancy could be due to other factors such as the original bacterial load in the urine and the quality of the sample tested. Nevertheless, it is important to highlight that only $24 / 55$ sequences were interpretable, indicating the limitations of this method.

An interesting result was that many of the sequence types were not similar to any of the known reference strains. Although $\sec Y$ sequences of all the known reference strains are not listed in Genbank, this finding suggests that new local strains have yet to be identified and reinforces the need for further epidemiological studies in Brazil and other tropical regions. While very useful, sequencing of PCR products cannot, as yet, replace the need for bacterial culture of leptospires. The need for identification of local isolates in tropical regions has been recently discussed [9] and genome sequencing of newly identified isolates will broaden our understanding of the circulating strains.

This study also demonstrates the presence of diverse species and genotypes of Leptospira infecting cattle in the same region. It is certainly possible that a particular subset of strains cannot be adequately classified by $\sec Y$, and this merits further study. Other studies using the $\sec Y$ sequencing method have also showed genetic diversity of Leptospira in human blood and animal specimens [5, 6]. The majority of $\sec Y$ sequence types displayed closest homology with strains isolated in Brazil compared to elsewhere. Two sequences were homologous to $L$. santarosai sv Bananal obtained from capybaras in São Paulo and, more recently, goats from Rio de Janeiro [9], but three sequence types were classified as $L$. interrogans and showed similarity with bovine strains from São Paulo, and Pelotas in Southern Brazil, perhaps indicating that relatively few genetic populations prevail in bovine leptospirosis in Brazil.

An unexpected finding was that almost half of the samples investigated were identified as L. borgpetersenii, which is consistent with the prevalence of this species in cattle in other countries. While $L$. interrogans Hardjoprajitno is mainly found in Europe, in other regions, such as Australia and New Zealand, L. borgpetersenii Hardjobovis most often prevails $[1,2,4]$. In Brazil, L. borgpetersenii Hardjobovis has never been isolated by culture, and only two strains of $L$. interrogans Hardjoprajitno have been described $[10,11]$. In the present study, $70 \%$ of the sequence types of $L$. borgpetersenii were closely related to Hardjobovis, two of them with a high similarity $(>99 \%)$ which suggests that this serovar may also be infecting Brazilian cattle. A possible reason for the non-isolation of $L$. borgpetersenii in Brazil may be due to their difficulty to culture [12]. 
In conclusion, although it cannot replace the need for bacterial culturing of leptospires, direct sequencing of PCR products of the $\sec Y$ gene is a valid and useful tool for epidemiological studies of these organisms as it has revealed genetic diversity and possibly new strains of Leptospira infecting Brazilian cattle.

\section{SUPPLEMENTARY MATERIAL}

For supplementary material accompanying this paper visit http://dx.doi.org/10.1017/S0950268815001363.

\section{ACKNOWLEDGEMENTS}

This study was supported by FAPERJ, CNPq and CAPES. W.L. and M.A.M. are CNPq fellows. The authors thank Professor Odir Dellagostin (UFPel) and Professor Silvio Vasconcellos (USP) for providing local leptospiral strains.

\section{DECLARATION OF INTEREST}

None.

\section{REFERENCES}

1. Salgado M, et al. A cross sectional observational study to estimate herd level risk factors for Leptospira spp. serovars in small holder dairy cattle farms in southern Chile. BMC Veterinary Research 2014; 10: 126.

2. Fang $\mathbf{F}$, et al. Shedding and seroprevalence of pathogenic Leptospira spp. in sheep and cattle at a New Zealand abattoir. Zoonoses and Public Health 2015; 62: 258-268.

3. Dhaliwal GS, et al. Effect of Leptospira interrogans serovar hardjo infection on milk yield in endemic- ally infected dairy herds. Veterinary Record 1996; 139: 319-320.

4. Rinehart CL, et al. Efficacy of vaccination of cattle with the Leptospira interrogans serovar hardjo type hardjoprajitno component of a pentavalent Leptospira bacterin against experimental challenge with Leptospira borgpetersenii serovar hardjo type hardjo-bovis. American Journal of Veterinary Research 2012; 73: 735-740.

5. Bourhy $\mathbf{P}$, et al. Serovar diversity of pathogenic Leptospira circulating in the French West Indies. PLoS Neglected Tropical Diseases 2013; 7: 2114.

6. Perez J, Goarant C. Rapid Leptospira identification by direct sequencing of the diagnostic PCR products in New Caledonia. BMC Microbiology 2010; 10: 325.

7. Victoria B, et al. Conservation of the S10-spc-alpha locus within otherwise highly plastic genomes provides phylogenetic insight into the genus Leptospira. PLoS ONE 2008; 3: 2752.

8. Ahmed N, et al. Multilocus sequence typing method for identification and genotypic classification of pathogenic Leptospira species. Annals of Clinical Microbiology and Antimicrobials 2006; 23: 28-44.

9. Lilenbaum W, et al. Molecular characterization of the first leptospires isolated from goats in Brazil. Brazilian Journal of Microbiology 2014; 45: 1527-1530.

10. Cosate MR, et al. Molecular characterization by LSSP-PCR and DNA sequencing of a pathogenic isolate of Leptospira interrogans from Brazil. Zoonoses and Public Health 2012; 59: 379-388.

11. Director A, et al. Isolation of Leptospira interrogans Hardjoprajitno from vaginal fluid of a clinically healthy ewe suggests potential for venereal transmission. Journal of Medical Microbiology 2014; 63: 1234-1236.

12. Bulach DM, et al. Genome reduction in Leptospira borgpetersenii reflects limited transmission potential. Proceedings of the National Academy of Sciences USA 2006; 103: 14560-14565. 